\title{
Laryngeal squamous cell carcinoma variants: clinical and pathological analysis of 110 patients over a 10-year period
}

\author{
ibrahim yağc1 ${ }^{1}$, Can Doruk ${ }^{2}$, and Bora Başaran ${ }^{2}$ \\ ${ }^{1}$ Istanbul Universitesi \\ ${ }^{2}$ Istanbul Universitesi Istanbul Tip Fakultesi
}

May 27, 2020

\begin{abstract}
Objectives: Squamous cell carcinoma(SCC) is the most common laryngeal neoplasm. Squamous cell carcinoma variants (vSCC), on the other hand, show different clinical and pathological features than conventional type. The purpose of this study is to analyze the clinical and pathological findings of vSCC of the larynx. Methods: This retrospective study evaluates 110 patients diagnosed with vSCC in our institution between 2006-2017. Treatment of primary and recurrent diseases was evaluated. Overall survival, disease-specific survival and follow-up times were calculated as months. Difference between preoperative and postoperative pathology reports of surgically treated patients were compared. Results: There were 1497 patients diagnosed with laryngeal malignancy and $110(7,34 \%)$ of these patients were diagnosed with vSCC. The most common pathological subgroup was verrucous carcinoma. The best prognosis was found in verrucous carcinoma and the worst was in spindle cell carcinoma. Overall survival rates was $90 \%$ and $54.7 \%$, respectively. A group of patients had a preoperative pathological diagnosis, not vSCC, but conventional SCC with the incidence of $38.5-100 \%$ according to the histological subtype. This was most common in acantholytic carcinoma, followed by adenosquamous, basaloid and spindle cell carcinoma, respectively. Conclusions: Spindle cell carcinoma and basaloid type squamous cell carcinoma have the worst prognosis and the highest metastatic potential. Patients diagnosed with these two variants should be followed up more cautiously. It should also be kept in mind that the diagnosis of vSCC can be missed in patients diagnosed only with a small tissue biopsy sample.
\end{abstract}

\section{MAIN TEXT:}

\section{Introduction}

Laryngeal malignancies constitute $1 \%$ of overall and $13 \%$ of the head and neck region malignancies. [1] Principally, laryngeal malignancies are classified into 2 main groups according to the histopathological characteristics: Squamous cell carcinoma (SCC) and non-SCC laryngeal malignancies.[2,3] Also SCC is classified into two groups according to the pathological features: conventional (classic) type and variants of SCC (vSCC), whereas vSCC constitutes up to $5 \%$ of all laryngeal malignancies.[3]

Even though there is vast knowledge present in the literature about the classification and clinical properties of classical type SCC, limited information about the vSCC challenges physicians during the treatment.[4] This study aims to provide a clinical and pathological evaluation of vSCC with a literature review. Our secondary aim is to compare the preoperative and postoperative pathological results of the patients with laryngeal SCC.

\section{Materials and Method}

The Ethics Committee's approval of this study was taken from/removed for blind peer review/ Ethics Committee with the file number of $2018 / 716$. 
In this study, all patients who underwent open or transoral laser surgery for laryngeal malignancies or biopsied for the suspicion of a laryngeal malignancy in a tertiary university hospital's Ear Nose Throat and Head and Neck Surgery clinic between the years 2006 - 2017 were evaluated.

All patients with pathology result other than vSCC (conventional SCC and non-SCC laryngeal malignancies) were excluded from the study. A retrospective chart analysis was made for all patients with a definite vSCC pathology report including information of sex, age at the time of diagnosis, localization of the primary lesion, TNM stage at the time of diagnosis, primary treatment modality (surgery, radiotherapy (RT) or chemoradiotherapy (CRT), adjuvant treatment modalities if available and cause of death for the patients who died during the follow-up. Overall survival, disease-free survival and overall follow-up period was calculated as months.

Lastly, preoperative and postoperative pathology reports of surgically treated patients were compared to analyze whether a difference exists between preoperative results from small biopsy specimens and definite pathology reports form the excision material.

Microsoft Excel Volume 15.31 (170216) and SPSS 21.0 programs were used for the statistical analysis of the study. Kaplan - Meier analysis was used for the survival analysis.

\section{Results}

Overall, 1497 patients who were diagnosed with a laryngeal malignancy after a diagnostic or curative surgery, were identified between January 2006 and January 2018. The mean age of the overall patients was 60,3 $\pm 10,35(32-95)$, and $92.2 \%$ of the patients were male (M/F: 1380/117). Overall, $89.9 \%(1347 / 1497)$ of the pathology reports were classic (conventional) type SCC, and \%2.7 (40/1497) were non-SCC laryngeal malignancies.

The remaining $110(7.34 \%)$ patients who were diagnosed with vSCC were included to study. The average age at the time of diagnosis of this group was $61,6 \pm 9,65(30-84)$. While $92 \%$ of the patients were male, only $8 \%$ were female $(\mathrm{M}: \mathrm{F}=101: 9)$. Median follow-up duration was 48 months (1-158)

All patients with a primary tumor stage of T3 and more with no previous radiotherapy history (for other malignancies than laryngeal carcinoma) underwent elective neck dissection according to the localization and lateralization of the tumor. No neck dissection was performed for patients whose preoperative diagnosis was verrucous carcinoma. For patients with a T1 and T2 tumor stage, elective neck dissection was performed only if clinical and radiological metastasis presence or occurrence was present.

Demographic information, tumor localizations, TNM stages and overall stages of the patients with vSCC is given in detail in Table 1and follow-up durations and survival information in Table 2

\section{Verrucous Carcinoma}

Overall, 34 patients were diagnosed with verrucous carcinoma, and while the primary treatment modality was surgery in 32 patients, the remaining 2 patients were treated with primary RT due to patient preferences. During the follow-up period, salvage total laryngectomy was performed in one of the patients due to recurrent disease, while the other patient was followed up as disease-free. The information about the treatment modalities of the primary disease, recurrence, and treatment of recurrence is given in Table 3 .

During the follow-up, 6 patients were lost due to systemic diseases not related to laryngeal malignancy: Cerebrovascular Accident, hepatocellular carcinoma and chronic obstructive pulmonary disease (COPD). The median follow-up duration was 84 months. 5-years disease-specific survival is $100 \%$ and overall survival is $90 \%$.

\section{Basaloid SCC}

Overall, 28 patients were diagnosed with basaloid carcinoma, and while the treatment modality was surgery in 25 patients, the remaining 3 patients were treated with primary RT or CRT. The information about the treatment modalities of the primary disease, recurrence, and treatment of recurrence is given in Table 3 . 
During the follow-up, a second primary lung malignancy was detected in 3 patients all of whom were treated with surgery and CRT for larynx, and all of them died due to the lung malignancy. One patient died during primary CRT and one during adjuvant CRT due to accompanying medical problems. One patient died for a reason not related to the disease. Lastly, 5 patients died due to tumor recurrence. The remaining 17 patients were disease-free in their last follow-up.

The median follow-up duration is 57 months (8-141). 5-years disease-specific and overall survival rates are $69.9 \%$ and $61.8 \%$ respectively.

Spindle cell SCC

Overall, 16 patients were diagnosed with spindle cell variants, and 15 were treated with primary surgery and one patient with CRT. The information about the treatment modalities of the primary disease, recurrence, and treatment of recurrence is given in Table 3 .

During the follow-up, 7 patients were lost due to local or systemic recurrence of the disease in the median 4 months (1-18).

5 -years disease-specific and the overall survival rate is $54.7 \%$.

Acantholytic SCC

Overall, 17 patients were diagnosed with acantholytic variants of SCC. Sixteen of the patients were treated with primary surgery and one with primary RT. During the follow-up of the patient who had primary radiotherapy, recurrence was detected, and salvage total laryngectomy was performed. The patient is followed up disease-free. The information about the treatment modalities of the primary disease, recurrence, and treatment of recurrence is given in Table $\mathbf{3}$.

Five of the patients were lost due to locoregional or systemic recurrence of the primary disease within the median 13 months (5-69). Furthermore, 2 patients died during the $6^{\text {th }}$ and $12^{\text {th }}$ years of the follow-up due to medical conditions other than primary disease. The remaining 9 patients were disease-free at their last follow-up.

The median follow-up duration is 30 months (5-158). 5-years disease-specific and overall survival rates are $72,8 \%$ and $60,7 \%$ respectively.

\section{Adenosquamous Carcinoma}

In total 9 patients were diagnosed with the adenosquamous variant of SCC, and for all the patients, surgery was the primary treatment method. In one patient neck dissection was not performed due to previous neck irradiation history for nasopharyngeal carcinoma. The information about the treatment modalities of the primary disease, recurrence, and treatment of recurrence is given in Table 3 .

During the follow-up, one patient was lost due to cerebrovascular accident and its related complications and one due to systemic recurrence of the primary disease. The remaining 7 patients were disease-free at the last follow-up.

The median follow-up duration is 61 months (17-144). 5-years disease-specific survival is $\% 83,3 \%$ and the overall survival is $72,9 \%$.

\section{Lymphoepithelial Carcinoma}

Overall, 4 patients were diagnosed with lymphoepithelial carcinoma variants. All of the patients were surgically treated. The information about the treatment, recurrence and treatment of recurrence is given inTable 3 .

In one patient systemic metastasis occurred at the early follow-up period, and the patient was lost in the postoperative $9^{\text {th }}$ month despite chemotherapy treatment. One patient died for a reason not related to the disease. The remaining 2 patients were disease-free at their last follow-up. 
The median follow-up duration is 39 months (9-119). 5-years disease-specific and overall survival rate is $75 \%$. Papillary SCC

Only 2 patients were diagnosed with papillary variants of SCC. One of the patients was treated with surgery while primary CRT was applied for the other patient, for whom conservation surgery was not possible.

Due to the tumor stage and primary treatment modalities, in none of the patients, elective neck dissection was performed. The patients were disease-free at their last follow-ups which were in the $17^{\text {th }}$ and $28^{\text {th }}$ months, respectively. Due to the short follow-up period, 5-year survival for papillary variant SCC could not be calculated. One-year disease-specific and overall survival rate is $100 \%$.

\section{Comparison of preoperative and postoperative pathology report results:}

It was determined that in some cases even though the preoperative pathology result was conventional SCC, the final result was a vSCC. We have determined that $66 \%, 100 \%, 53.3 \%$ and $38.5 \%$ of the preoperative pathology reports were conventional SCC in patients with adenosquamous, acantholytic, basaloid and spindle cell carcinoma, respectively (Figure 1 ). For other variants (lymphoepithelial, papillary and verrucous carcinoma), a difference between primary and final pathology reports was not observed. Furthermore, no pathological subgroup changes after surgical treatement were observed in any patient diagnosed with primary vSCC.

\section{Discussion}

Laryngeal malignancies almost always arise from the surface epithelium and thus, more than $95 \%$ are squamous cell carcinoma.[5] Similarly, our results are consistent with the literature with an incidence of $90 \%$ conventional SCC, $7.3 \%$ vSCC and 2.7 non-SCC laryngeal malignancies.

Verrucous carcinoma is the most common variant of SCC both in the literature and in our series. In a review published in 2016, the incidence of laryngeal verrucous SCC was reported to be 1-3.4\%.[6] In our study, the incidence of verrucous carcinoma within the entire laryngeal malignancies is calculated as $2.3 \%$ $(34 / 1497)$, which is similar to the literature. Verrucous carcinoma mostly arises from the glottic region and hence frequently can be diagnosed at the early stages.[7] Furthermore, regional or distant metastasis is not common. For these reasons, transoral laser surgery is often adequate with excellent survival rates.[7] In the present study, $73 \%$ of the patients had a T1a tumor, and $88 \%$ of tumors were localized in the glottic region with an overall 5-years survival rate of $100 \%$. No neck and distant metastasis occurred in any of the patients in the study group, thus for patients with pre-operative verrucous carcinoma diagnosis, elective neck dissection is not necessary.

Laryngeal basaloid SCC is an aggressive variant of SCC which was first described in 1986.[8,9] Laryngeal basaloid SCC is often diagnosed in the supraglottic localization. Begum et al. detected the tumor in the supraglottic localization in all of the patients (8/8)[10], and Ereno et al in 86\% (13/15).[11] In our patient group, the supraglottic region is the most common localization of the tumor (82\%). Published studies indicated that neck metastasis occurs up to $50-70 \%$ in patients with basaloid SCC.[12,13] In our study, neck metastasis was observed in $46.4 \%$ of the patients. Due to the higher tendency to regional and distant metastasis, lower survival rates have been published. In a study published in 2008, 3 and 5-year overall survival of 15 patients reported as $50 \%$ and $38.5 \%$, respectively.[11] In our study, 5-year disease-specific survival was determined as $69.9 \%$. While the survival rate is $100 \%$ for the early stages, a decline to $54.5 \%$ is observed for late-stage tumors.

Spindle cell carcinoma was first described by Virchow in 1863 and in 1933 Figi described the entire pathological details.[14] The most common origin of the spindle cell carcinoma is the larynx.[15] Even though the prognosis is directly related to the stage, spindle cell carcinoma is an aggressive tumor.[14] In the present study, according to the survival rates, spindle cell carcinoma is the variant with the worst prognosis.

Laryngeal papillary SCC was first described in 1999 by Thompson et al.[16] including 104 cases as a variant of SCC with a good prognosis. Similar to the literature, survival rates are also excellent in our study. 
Even though, our follow-up period is inadequate to give 5-years survival rates, both of our patients are disease-free at their $17^{\text {th }}$ and $28^{\text {th }}$-month follow-up. In a study in 2015,370 patients were evaluated by using the Surveillance, Epidemiology, and End Results (SEER) Program, and the overall incidence within entire laryngeal malignancies was calculated as 0.5\%.[17] In the present study, an incidence of $0.1 \%$ was calculated. High-risk Human Papilloma Virus (HPV) strains are thought to play a role in the etiology of the papillary variant of SCC. $[18,19]$ Our lower rates can be explained by the low incidence of these strains in our country.[20] Due to the retrospective nature of our study and lack of HPV-DNA or p16 screening, we can't make a definite interpretation.

Laryngeal lymphoepithelial carcinoma is a variant of SCC that has a resemblance to undifferentiated nonkeratinized (WHO type 3) nasopharyngeal carcinoma and a high affinity to neck metastasis.[5,21] Similar to the literature, neck metastasis was observed in $75 \%$ of our patient group. According to SEER data in which 16 patients were evaluated, overall and disease specific 5-years survival of this variant was $58,8 \%$ and $63 \%$, respectively.[22] The overall and disease specific 5-year survival in our study was assessed as $50 \%$ and $75 \%$, which was slightly better compared to this study. However, due to our low number of patients (4), a comparison with the literature would not be suitable.

Acantholytic SCC was classified as a vSCC until 2017, however, after 2017 this variant was included in the conventional laryngeal SCC group. $[5,23]$ The evaluation of the patients in this study group was done with the previous classification system, thus 17 patients were diagnosed with the acantholytic variant. Overall 5 -years survival was calculated as $72.8 \%$ and neck metastasis was observed in $31.3 \%$ of the patients who were treated with primary surgical methods. Our results show similarity to prognosis and neck metastasis rates of the classical laryngeal SCC.[24,25]

Laryngeal adenosquamous carcinoma is a vSCC with both glandular and epithelial differentiation. Previous studies of this variant show neck metastasis up to $75 \%$ and distant metastasis up to $25 \%$ of the cases. [5] Adenosquamous carcinoma is the vSCC with one of the worst prognosis, and previous 5-years survival studies show around 30-50\% survival rates.[5] Controversially, in our study, the 5-years survival rate for adenosquamous carcinoma is $83.3 \%$. The explanation for this great difference can be explained by the absence of any patients with distant metastases at the time of diagnosis in this pathological subgroup, which will also be mentioned in the limitation of the study. Due to the absence of this feature, which is a bad prognostic factor, better survival rates were calculated.

We found that some of the patients included in this study had a preoperative pathological diagnosis, not vSCC, but conventional SCC with the incidence of 38.5-100\% according to the histological subtype. It is possible to detect pathological subgroup changes in the operated patients, but it is not possible to detect this situation in the patient group who underwent non-surgical treatment. For this reason, several patients who should be in the vSCC group are considered as classic SCC, and treatment and follow-up planning can be made with this information. Another result of this condition is the survival data of our study; the survival data of some pathological subgroups were observed better than the literature. One of the possible causes of this condition may be that the patients with poor prognostic indicators, inoperable tumors and distant metastases receive non-surgical treatments, and the diagnosis of these patients should be reported as conventional SCC, not vSCC. Thus, the patient group in our study may have consisted of patients with a relatively good prognosis.

The difference between the diagnostic and definite pathology results can be explained by the heterogeneity of the tumors, which creates areas that do not contain the vSCC. Although smaller specimens are adequate for the malignity diagnosis, sub-typing is not always possible. A small biopsy is usually taken for diagnosis of laryngeal cancer. Because large biopsy fragments can cause bleeding, edema, and associated airway obstruction. In patients with small lesions planned to be treated with primary RT, permanent vocal cord damage and permanent quality of voice may occur after treatment. It can be concluded from this study that small punch biopsies may not be sufficient for the diagnosis of vSCC.

While evaluating the patients for adjuvant therapies, along with the TNM stage of the tumor, pathological 
subgroup change possibility should also be taken into account. Especially, the worse prognosis and high metastatic potentials of spindle cell carcinoma and basaloid type SCC should be kept in mind. Patients who are diagnosed with these two variants should be followed-up more carefully, and the physicians should be more skeptic if any recurrence suspicion is present.

The first limitation of our study is the retrospective design. The clinical and pathological staging of the tumors was reported by using the $7^{\text {th }}$ Edition of The American Joint Committee on Cancer (AJCC), which was renovated by the $8^{\text {th }}$ Edition in January 2018. Secondly, the vast majority of the patients who were included in the study are patients who were surgically treated. Thus, some of the patients with distant metastasis at the time of the diagnosis might be missed out. For this reason, the survival rates might be lower than our calculated results. Even though prospective studies can be designed for better prognosis and survival rate analysis, differences between pre-operative and definite pathology results will always cause under-diagnosis of variants.

\section{References}

[1] Bray F, Ferlay J, Soerjomataram I, Siegel RL, Torre LA, Jemal A. Global cancer statistics 2018: GLOBOCAN estimates of incidence and mortality worldwide for 36 cancers in 185 countries. CA Cancer J Clin 2018;68:394-424. https://doi.org/10.3322/caac.21492.

[2] Lewis JS. Not Your Usual Cancer Case: Variants of Laryngeal Squamous Cell Carcinoma. Head Neck Pathol 2011;5:23-30. https://doi.org/10.1007/s12105-010-0232-0.

[3] Thompson LDR. Laryngeal dysplasia, squamous cell carcinoma, and variants. Surg Pathol Clin 2017;10:15-33.

[4] Lin HW, Bhattacharyya N. Staging and survival analysis for nonsquamous cell carcinomas of the larynx. Laryngoscope 2008;118:1003-13.

[5] El-Naggar AK, Chan JKC, Grandis JR, Takata T, Slootweg PJ. WHO classification of head and neck tumours. 4th ed. International Agency for Research on Cancer; 2017.

[6] Echanique KA, Desai S V., Marchiano E, Spinazzi EF, Strojan P, Baredes S, et al. Laryngeal Verrucous Carcinoma: A Systematic Review. Otolaryngol - Head Neck Surg (United States) 2017;156:38-45. https://doi.org/10.1177/0194599816662631.

[7] Dubal PM, Svider PF, Kam D, Dutta R, Baredes S, Eloy JA. Laryngeal verrucous carcinoma: a population-based analysis. Otolaryngol Neck Surg 2015;153:799-805.

[8] Wain SL, Kier R, Vollmer RT, Bossen EH. Basaloid-squamous carcinoma of the tongue, hypopharynx, and larynx:: Report of 10 cases. Hum Pathol 1986;17:1158-66.

[9] Bahar G, Feinmesser R, Popovtzer A, Ulanovsky D, Nageris B, Marshak G, et al. Basaloid squamous carcinoma of the larynx. Am J Otolaryngol 2003;24:204-8.

[10] Begum S, Westra WH. Basaloid squamous cell carcinoma of the head and neck is a mixed variant that can be further resolved by HPV status. Am J Surg Pathol 2008;32:1044-50. https://doi.org/10.1097/PAS.0b013e31816380ec.

[11] Ereño C, Gaafar A, Garmendia M, Etxezarraga C, Bilbao FJ, López JI. Basaloid squamous cell carcinoma of the head and neck: A Clinicopathological and Follow-Up Study of 40 Cases and Review of the Literature. Head Neck Pathol 2008;2:83-91. https://doi.org/10.1007/s12105-008-0045-6.

[12] Soriano E, Faure C, Lantuejoul S, Reyt E, Bolla M, Brambilla E, et al. Course and prognosis of basaloid squamous cell carcinoma of the head and neck: a case-control study of 62 patients. Eur J Cancer 2008;44:24450.

[13] Calhoun KH, Fulmer P, Weiss R, Hokanson JA. Distant metastases from head and neck squamous cell carcinomas. Laryngoscope 1994;104:1199-205. 
[14] Dubal PM, Marchiano E, Kam D, Dutta R, Kalyoussef E, Baredes S, et al. Laryngeal spindle cell carcinoma: A population-based analysis of incidence and survival. Laryngoscope 2015;125:2709-14.

[15] Gerry D, Fritsch VA, Lentsch EJ. Spindle cell carcinoma of the upper aerodigestive tract: an analysis of 341 cases with comparison to conventional squamous cell carcinoma. Ann Otol Rhinol Laryngol 2014;123:576-83.

[16] Thompson LDR, Wenig BM, Heffner DK, Gnepp DR. Exophytic and papillary squamous cell carcinomas of the larynx: a clinicopathologic series of 104 cases. Otolaryngol Neck Surg 1999;120:718-24.

[17] Dutta R, Husain Q, Kam D, Dubal PM, Baredes S, Eloy JA. Laryngeal papillary squamous cell carcinoma: a population-based analysis of incidence and survival. Otolaryngol Neck Surg 2015;153:54-9.

[18] Halec G, Holzinger D, Schmitt M, Flechtenmacher C, Dyckhoff G, Lloveras B, et al. Biological evidence for a causal role of HPV16 in a small fraction of laryngeal squamous cell carcinoma. Br J Cancer 2013;109:172-83.

[19] Manjarrez ME, Ocadiz R, Valle L, Pacheco C, Marroquin A, De la Torre C, et al. Detection of human papillomavirus and relevant tumor suppressors and oncoproteins in laryngeal tumors. Clin Cancer Res 2006;12:6946-51.

[20] Sunter AV, Hamit B, Yigit O, Server EA, Omeroglu Kara E, Karatas A, et al. The Prevalence of Tonsillar Human Papilloma Virus Infection in Istanbul, Turkey: A Human Cadaver Study. Turkish Arch Otorhinolaryngol 2019;57:117-21. https://doi.org/10.5152/tao.2019.4312.

[21] Ibrahimov M, Yilmaz M, Celal MH, Mamanov M, Yollu U, Ozek H. Lymphoepithelial carcinoma of the larynx. J Craniofac Surg 2013;24:1049. https://doi.org/10.1097/SCS.0b013e3182700cd9.

[22] Chan JYK, Wong EWY, Ng S-K, Vlantis AC. Non-nasopharyngeal head and neck lymphoepitheliomalike carcinoma in the United States: A population-based study. Head Neck 2016;38:E1294-300. https://doi.org/10.1002/hed.24215.

[23] Barnes L, Tse L, Hunt J, Brandwein-Gensler M, Urken M. WHO Classification of Head and Neck Tumours 2007:107-31.

[24] Hoffman HT, Porter K, Karnell LH, Cooper JS, Weber RS, Langer CJ, et al. Laryngeal cancer in the United States: Changes in demographics, patterns of care, and survival. Laryngoscope 2006;116:1-13. https://doi.org/10.1097/01.mlg.0000236095.97947.26.

[25] Cosetti M, Yu GP, Schantz SP. Five-year survival rates and time trends of laryngeal cancer in the US population. Arch Otolaryngol - Head Neck Surg 2008;134:370-9. https://doi.org/10.1001/archotol.134.4.370.

\section{FIGURE LEGENDS:}

Table 1: Demographic data and distribution of tumor localization and tumor stages of the study group. (Abbreviations; SCC: squamous cell carcinoma, M: male, F: female, ND: Neck Dissection),

* All patients with a primary tumor stage of T3 and more with no previous radiotherapy (for other malignancies than laryngeal carcinoma) history underwent elective neck dissection according to the localization and lateralization of the tumor. No neck dissection was performed for patients whose preoperative diagnosis was verrucous carcinoma. For patients with a T1 and T2 tumor stage, elective neck dissection was performed only if clinical and radiological metastasis presence or occurrence was present.

Table 2: Median follow-up periods and overall and disease-specific survival rates for each variant, stage, and localization . (Abbreviations; n: number, OS: Overall Survival, DSS: Disease-Specific Survival, SCC: squamous cell carcinoma)

: Follow-up period for papillary carcinoma is less than 5 years, 5 -year survival rates are not available. 
Table 3: T stages of the tumor, treatment methods, recurrence information and treatment methods for recurrence.

(Abbreviations; n: number, TOLS: Transoral Endolaryngeal Laser Surgery, TL: Total Laryngectomy, TLP: Total Laryngopharyngectomy sTL: Salvage Total Laryngectomy, nTL: Near-Total Laryngectomy SGLpF: Supraglottic Laryngectomy and Partial Pharyngectomy, VHL: Vertical Hemilaryngectomy, SGL: Supraglottic Laryngectomy, SCL: Supracricoid laryngectomy, ND: Neck dissection, RT: Radiotherapy, CRT: Chemoradiotherapy, DOD: died of disease, NED: no evidence of disease )

Figure 1 : Distribution of patients diagnosed with vSCC but with a preoperative pathological diagnosis of conventional SCC.

\section{Hosted file}

@Table 1.docx available at https://authorea.com/users/326799/articles/454569-laryngealsquamous-cell-carcinoma-variants-clinical-and-pathological-analysis-of-110-patientsover-a-10-year-period

\section{Hosted file}

@Table 2.docx available at https://authorea.com/users/326799/articles/454569-laryngealsquamous-cell-carcinoma-variants-clinical-and-pathological-analysis-of-110-patientsover-a-10-year-period

\section{Hosted file}

@Table 3.docx available at https://authorea.com/users/326799/articles/454569-1aryngealsquamous-cell-carcinoma-variants-clinical-and-pathological-analysis-of-110-patientsover-a-10-year-period

\section{Hosted file}

QFigure 1.docx available at https://authorea.com/users/326799/articles/454569-1aryngealsquamous-cell-carcinoma-variants-clinical-and-pathological-analysis-of-110-patientsover-a-10-year-period 